\title{
Comparative Analysis in Terms of Computational Cost for Different Discrimination Algorithms in Implantable Defibrillators
}

\author{
A Cebrian ${ }^{1}, \mathrm{~J} \mathrm{Rey}^{1}, \mathrm{~J} \mathrm{Millet}^{1}$, JJ Rieta ${ }^{1}, \mathrm{R}_{\text {Garcia-Civera }}{ }^{2}$ \\ ${ }^{1}$ Department of Electronics Engineering, Polytechnic University of Valencia, Spain \\ ${ }^{2}$ Electrophysiology Laboratory, University Clinic Hospital of Valencia, Spain
}

\begin{abstract}
Implantable Defibrillators (ICDs) use very low computational cost criteria (rate, stability and onset) offering good sensitivity for arrhythmia detection. Although, the specificity of these combined criteria decreases in difficult arrhythmia discrimination as in case of discrimination between Ventricular Tachycardia (VT) and Supraventricular Tachycardia (SVT).

Several morphological published algorithms enhance arrhythmia discrimination but most algorithms are developed in personal computers and cannot be used in ICDs because of computational cost requirements compared with limited ICD capabilities. A general method to determine the possibility of ICD implementation for a discrimination algorithm has been proposed.
\end{abstract}

\section{Introduction}

The classic criteria used in Implantable Defibrillators (ICDs) based on rate, stability and sudden onset calculations over electrogram (EGM), offer good sensitivity for arrhythmia detection. The requirements of these combined criteria in terms of computational cost are very low and can be implemented in ICDs without problems.

Although the good sensitivity of these combined criteria, specificity decreases in difficult arrhythmia discrimination as in case of discrimination between Ventricular Tachycardia (VT) and Supraventricular Tachycardia (SVT) [1].

In these difficult cases, ICD specificity can be increased using morphological criterion based algorithms. In fact, several morphological published algorithms that enhance arrhythmia discrimination can be found [2-5]. But most of these published algorithms are developed in personal computers and cannot be used in ICDs because of computational cost requirements compared with limited ICD capabilities.

A general method to determine the possibility of ICD implementation for a discrimination algorithm will be proposed. A quantitative overload ratio will be calculated in order to determine the ICD portability of a given arrhythmia discrimination algorithm.

\section{Discrimination algorithms}

Three morphological published discrimination algorithms with increasing complexity have been selected in order to test the proposed method to determine the possibility of ICD implementation:

- $\quad$ Time domain (complex peak area comparison) [2].

- $\quad$ Simplified Wavelet [3, 4].

- Frequency domain (Fourier complex spectrum analysis) [5].

Each algorithm has been reconstructed from author published information without access to original algorithm implementation.

\section{TIME DOMAIN}

This discrimination algorithm differentiates between VT and SVT by analysis of ventricular complexes morphology on a beat-to-beat basis in time domain.

The algorithm compares a test complex to a previously stored complex template in baseline rhythm. The comparison is accomplished calculating the difference between the peak areas of the test and template complexes. The two complexes are aligned and the area of each peak is normalized (figure 1).

The differences between the calculated peak areas are summed:

$$
\text { area_dif }=\mid \text { area of } \mathrm{A}-\text { area of } \mathrm{A}^{\prime} \mid+
$$

$\mid$ area of $\mathrm{B}-$ area of $\mathrm{B}^{\prime}|+|$ area of $\mathrm{C}-$ area of $\mathrm{C}^{\prime} \mid$

A template percent match score is inversely proportional to these differences:

$$
\operatorname{Match}(\%)=\frac{1}{\text { area_dif }} \cdot 100
$$

If the template percent match score is greater than a programmable threshold (range $30 \%$ to 95\%) for a number of programmable complexes, the discrimination algorithm indicates SVT otherwise VT. 


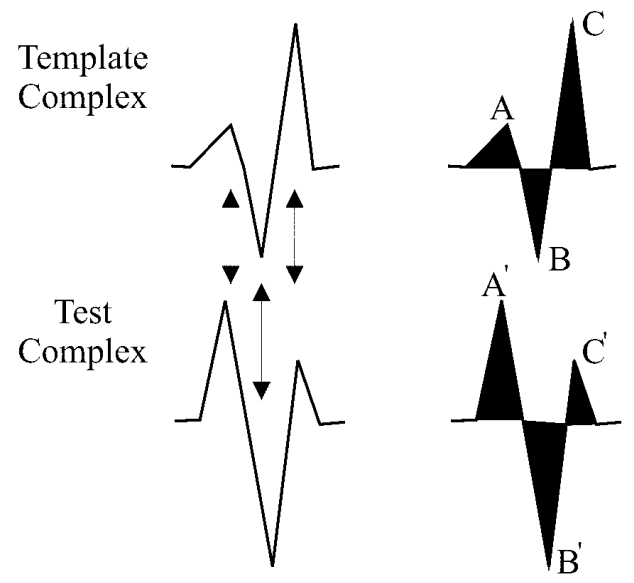

Figure 1. Complex peak area comparison algorithm

\section{SIMPLIFIED WAVELET}

This discrimination algorithm differentiates between VT and SVT by analysis of ventricular tachycardia electrograms using the Wavelet transform. The algorithm is based in the fact that alteration on the morphology of the complexes, in general, is because of the change in the origin chamber.

The algorithm compares the morphology of ventricular electrograms during a tachycardia with a template of electrogram morphology recorded during baseline rhythm using the Wavelet transform. A percent match score describes the degree of morphology similarity of the baseline and tachycardia electrograms (figure 2).

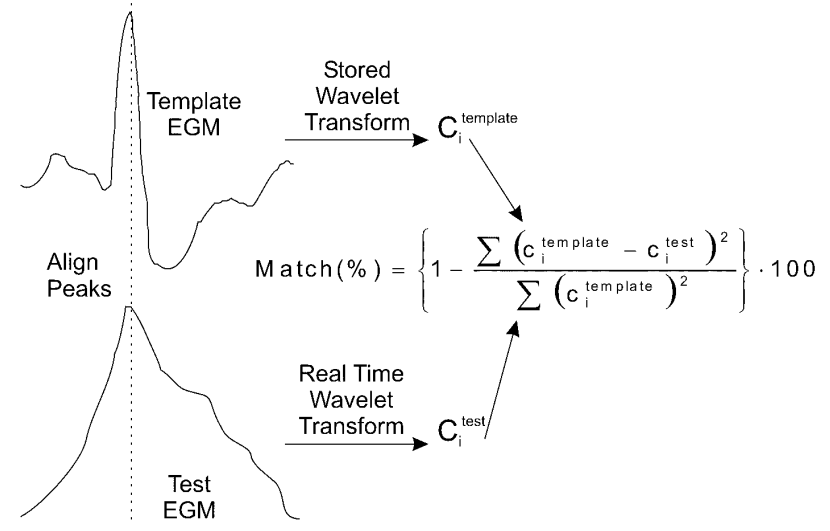

Figure 2. Simplified Wavelet based match score calculation

If the percent match score is below than $70 \%$ for a number of programmable complexes (at least 6 of 8), the tachycardia is classified as ventricular in origin (VT). Otherwise, the tachycardia is classified as atrial in origin (SVT).

Four steps are involved in the execution of the algorithm:

1. Wavelet transform of template electrogram (recorded during baseline rhythm) is calculated and stored.

2. Ventricular tachycardia electrograms, previous to rate criterion detection, are aligned with template electrogram in time domain (peak alignment).

3. Wavelet transform of each ventricular electrogram (test EGM) is calculated in real time.

4. The percent match score is calculated for each test EGM. This score is based on the differences between the test and template electrograms Wavelet transform coefficients.

Some simplifications have been adopted in the algorithm implementation to reduce computational requirements and enable real time processing:

- Haar Wavelet transform has been used because they simplify computation.

- The original Haar Wavelet transform has been modified defining the amplitude of all Wavelet functions to be either 1 or -1 . Using these amplitude values no multiplications are needed, reducing the computational cost of the Wavelet transform implementation.

- All calculated Wavelet coefficients smaller in absolute value than a programmed threshold are removed. This reduces noise and decreases computational cost.

\section{FREQUENCY DOMAIN}

The last discrimination algorithm differentiates between rhythms originated in the ventricle, such as ventricular fibrillation (VF) and ventricular tachycardia (VT), and rhythms originated in the atria, such normal sinus rhythm (SR) and supraventricular tachycardia (SVT). The algorithm is based in the alterations observed in the spectrum of the complexes (frequency domain) due to morphologic changes between ventricular and atrial rhythms.

When the rhythm is originated in the atria and travels through the normal conduction path, the complex spectrum contains high frequency components. When the rhythm is originated in the ventricle, the high frequency components of the complex spectrum are attenuated.

For the supraventricular rhythms (SR, SVT), the frequency spectrum has their maximum around $8 \mathrm{~Hz}$ and decrease slowly. While for ventricular rhythms (VT, VF), the spectrum is centered in lower frequencies, around $4 \mathrm{~Hz}$, and decreases quickly when the frequency increases.

Two steps are involved in the execution of the algorithm:

1. The complex spectrum is calculated using a discrete Fourier transform (DFT). Only five coefficients are calculated corresponding to 4,8 , 
12, 16 and $20 \mathrm{~Hz}$ spectral components.

2. The five calculated spectral components are introduced in a neural network to classify the complex as ventricular or atrial.

The neural network (figure 3) has one input layer composed of 5 neurons, a hidden layer of 4 neurons and an output layer of 1 neuron. The original implementation uses an output layer of 2 neurons but, for comparison reasons, the discrimination between VT and VF has been removed.

The neural network has been defined and trained using Matlab Neural Network Toolbox [6].

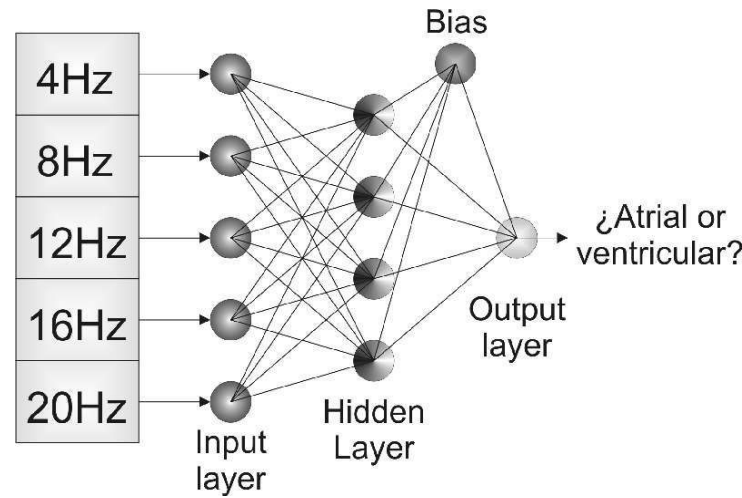

Figure 3. Fourier complex spectrum classification using a neural network

When the output of the neural network has a value near to 0 , the rhythm is classified as atrial. Otherwise, when the output is near to 1 the rhythm is classified as ventricular.

\section{Methods}

Remember that each algorithm has been reconstructed from author published information without access to original algorithm implementation. Because of this, differences between original and reconstructed algorithm can be found.

The reconstructed algorithm is implemented in MATLAB for testing purposes. After testing the reconstructed algorithm, the MATLAB implementation is ported to $\mathrm{C}$ language for computational cost estimation. Although, assembler language programming produces better code in terms of optimization, C language programming has been chosen to make migration to alternative target platforms easier.

The Texas Instruments MSP430 ultralow-power microcontroller family [7] has been chosen as the target platform for compilation purposes. The MSP430 architecture is optimized to achieve extended battery life in portable measurement applications. Its powerful 16-bit RISC CPU is optimized for maximum code efficiency, incorporating features specifically designed for the use of high-level languages such as $\mathrm{C}$.

The $\mathrm{C}$ language algorithm implementation is compiled for the MSP430 target using maximum optimization for speed. The compiler creates assembler output listing and instructions number $\left(\mathrm{N}_{\mathrm{ins}}\right)$ can be counted in order to determine the cycle number $\left(\mathrm{N}_{\text {cycles }}\right)$ through the cycles per instruction (CPI) conversion constant:

$$
\mathrm{N}_{\text {cycles }}=\mathrm{N}_{\text {ins }} \cdot \mathrm{CPI}
$$

But this is not an easy task because in the MSP430 $\mathrm{CPU}$, the cycles per instruction required for an instruction is not a constant and depends on the instruction format and the addressing mode used. Fortunately, the total cycle number can be calculated directly using the cycle counter feature of the MSP430 simulator/debugger (figure 4).

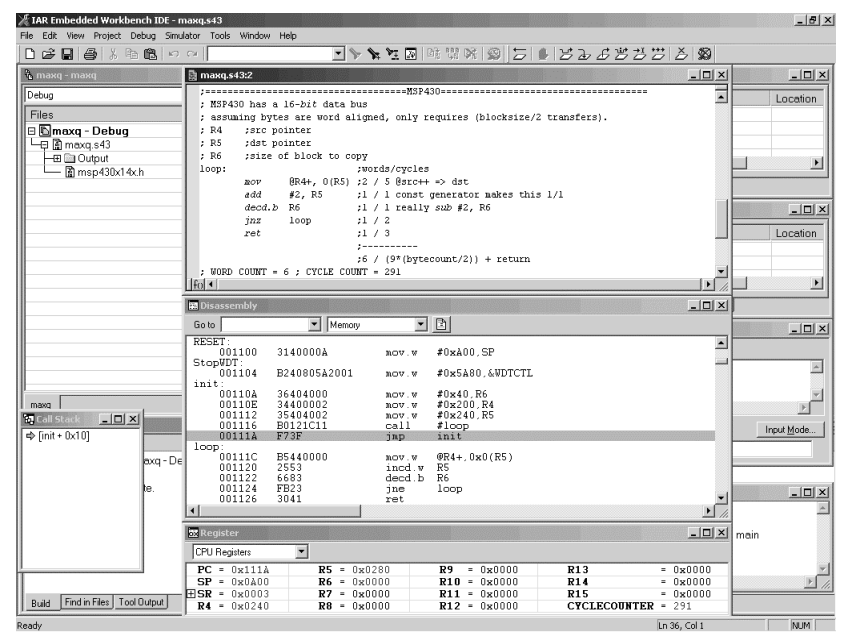

Figure 4. MSP430 simulator/debugger CYCLECOUNTER feature

The maximum allowed cycles that can be executed in the available discrimination time $\left(\mathrm{T}_{\mathrm{dis}}\right)$ at a $\mathrm{CPU}$ clock frequency $\left(\mathrm{f}_{\text {clock }}\right)$, can be obtained by the expression:

$$
\mathrm{N}_{\text {max_cycles }}=\mathrm{T}_{\text {dis }} \cdot \mathrm{f}_{\text {clock }}
$$

Total algorithm cycles calculated are compared with maximum allowed cycles to obtain an overload ratio:

$$
\mathrm{N}_{\text {max_cycles }}=\mathrm{T}_{\text {dis }} \cdot \mathrm{f}_{\text {clock }}
$$

Algorithms with an overload ratio below $100 \%$ can be implemented in the ICD. In other words, algorithms with an overload ratio below $100 \%$ can be executed in the available discrimination time running at the selected clock frequency.

\section{Results}

Assuming a CPU clock frequency of $32.768 \mathrm{kHz}$ and 5 seconds available for discrimination, the maximum allowed cycles are 163840 .

The three selected morphological discrimination 
algorithms have been analyzed with the proposed method to determine the possibility of ICD implementation:

\begin{tabular}{|c|c|c|c|}
\hline Algorithm & $\mathrm{N}_{\text {cycles }}$ & $\begin{array}{c}\text { Overload } \\
\text { Ratio (\%) }\end{array}$ & $\begin{array}{c}\text { Execution } \\
\text { time (s) }\end{array}$ \\
\hline \hline $\begin{array}{c}\text { Time domain } \\
\text { (complex peak area } \\
\text { comparison) }\end{array}$ & 24536 & 15 & 0.75 \\
\hline Simplified Wavelet & 60365 & 37 & 1.84 \\
\hline $\begin{array}{c}\text { Frequency domain } \\
\text { (Fourier complex } \\
\text { spectrum analysis) }\end{array}$ & 335519 & 205 & 10.24 \\
\hline
\end{tabular}

The comparative analysis (figure 5) of the calculated overload ratio for the selected discrimination algorithms shows that the three algorithms have an increasing complexity. Time domain (complex peak area comparison) and simplified Wavelet algorithms have an overload ratio below $100 \%$ and can be implemented in the ICD. Frequency domain (Fourier complex spectrum analysis) algorithm has an overload ratio greater than $100 \%$ and cannot be implemented in the ICD.

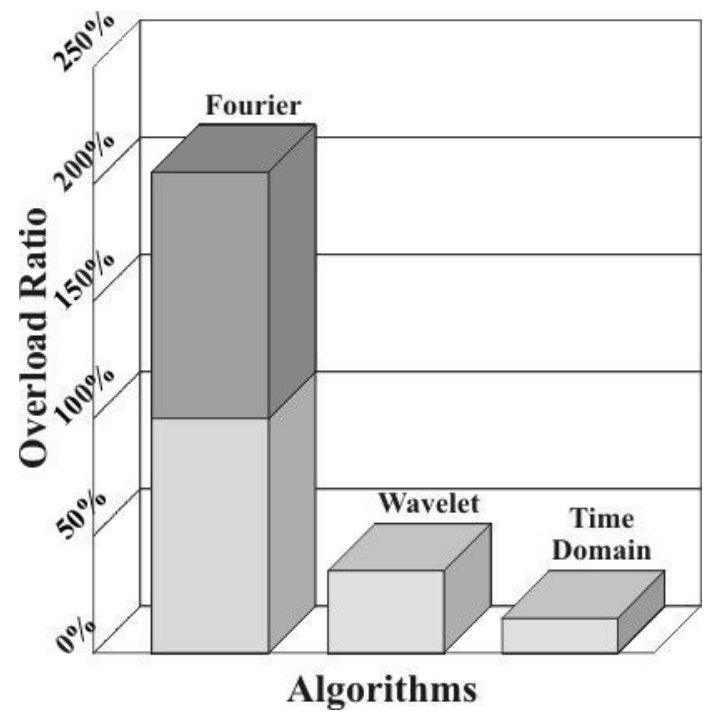

Figure 5. Overload ratio comparison for selected discrimination algorithms

\section{Conclusions}

A general method to determine the possibility of ICD implementation for a discrimination algorithm has been proposed attending to the algorithm computational cost requirements compared with ICD capabilities.
A quantitative overload ratio has been proposed to determine the ICD portability of a given arrhythmia discrimination algorithm. Algorithms with an overload ratio below $100 \%$ can be implemented in the ICD.

Three morphological published algorithms with increasing complexity have been selected: time domain (complex peak area comparison), simplified Wavelet and frequency domain (Fourier complex spectrum analysis) algorithms. Only Fourier analyzed algorithm has an overload ratio over $100 \%$ and cannot be implemented in an ICD.

\section{References}

[1] Charles D. Swerdlow, "Supraventricular TachycardiaVentricular Tachycardia Discrimination Algorithms in Implantable Cardioverter Defibrillators: State-of-the-Art Review", Journal of Cardiovascular Electrophysiology 2001 May;12(5):606-12

[2] Boriani G, Biffi M, Frabetti L, Lattuca JJ, Branzi A, "Clinical Evaluation of Morphology Discrimination: an Algorithm for Rhythm Discrimination in Cardioverter Defibrillators", Journal of Pacing and Clinical Electrophysiology 2001 Jun;24(6):994-1001

[3] Swerdlow CD, Brown ML, Lurie K, Zhang J, Wood NM, Olson WH, Gillberg JM, "Discrimination of Ventricular Tachycardia from Supraventricular Tachycardia by a Downloaded Wavelet-Transform Morphology Algorithm: A Paradigm for Development of Implantable Cardioverter Defibrillator Detection Algorithms", Journal of Cardiovascular Electrophysiology 2002 May;13(5):432441

[4] Koyrakh LA, Gillberg JM, Wood NM., "Wavelet transform based algorithms for EGM morphology discrimination for implantable ICDs", Computers in Cardiology 1999;343346.

[5] Kei-ichiro Minami, Hiroshi Nakajima, Takeshi Toyoshima, "Real-Time Discrimination of Ventricular Tachyarrhythmia with Fourier-Transform Neural Network", IEEE Transactions on Biomedical Engineering 1999 Feb;46(2):179-185

[6] Matlab Neural Network Toolbox: http://www.mathworks.com/products/neuralnet/?BB=1

[7] Texas Instruments, "MSP430x1xx Family User's Guide", 2004.

Address for correspondence

Antonio Cebrian

Universidad Politecnica de Valencia

Dept. de Ingenieria Electronica

Camino de Vera s/n

46022 Valencia (Spain)

acebrian@eln.upv.es 